\title{
Notes
}

\section{Structure Factor of a Semidilute Solution of Rodlike Macromolecules}

\section{PAUL VAN DER SCHOOT AND THEO ODIJK*}

Department of Polymer Technology, Faculty of Chemical Engineering and Materials Science, Delft University of Technology, P.O. Box 5045, 2600 GA Delft, The Netherlands

Received November 7, 1989;

Revised Manuscript Received February 5, 1990

Variational principles have been used extensively to solve integral equations occurring in the theory of polymer dynamics (see, e.g., refs 1-4). Similar principles of the Schwinger type can be applied to integral equations in the theory of scattering from semidilute and concentrated polymer solutions. Here we investigate such an application for rodlike macromolecules in the second virial approximation. The relevant integral equation has been introduced by Shimada et al. ${ }^{5,6}$ and is actually Yvon's equation for particles with orientational degrees of freedom. ${ }^{13}$ They solved this expression by a perturbation procedure, the convergence of which is unclear. ${ }^{5}$ Maeda solved the problem numerically. ${ }^{7}$ As an alternative we describe a simple variational treatment yielding a closed expression for the structure factor very close to the numerical data.

Doi et al. ${ }^{6}$ have used a variant of Yvon's theorem ${ }^{13}$ in order to calculate the structure factor $S(\mathbf{k})$ as a function of the wave vector $\mathbf{k}$

$$
\begin{gathered}
S(\mathbf{k})=\left\langle s_{\mathbf{k}}(\mathbf{u}) f(\mathbf{k}, \mathbf{u})\right\rangle \\
f(\mathbf{k}, \mathbf{u})+\frac{8 c}{\pi}\left\langle s_{\mathbf{k}}(\mathbf{u}) \sin \gamma\left(\mathbf{u}, \mathbf{u}^{\prime}\right) s_{\mathbf{k}}\left(\mathbf{u}^{\prime}\right) f\left(\mathbf{k}, \mathbf{u}^{\prime}\right)\right\rangle^{\prime}=s_{\mathbf{k}}(\mathbf{u}) \\
s_{\mathbf{k}}(\mathbf{u}) \equiv \frac{\sin \mathbf{K} \cdot \mathbf{u}}{\mathbf{K} \cdot \mathbf{u}} ; \quad \mathbf{K} \equiv \frac{1}{2} L \mathbf{k}
\end{gathered}
$$

Here, $\gamma$ is the angle between two test rods with orientations $\mathbf{u}$ and $\mathbf{u}^{\prime}$, the unit vectors along the respective long axes. The orientational average $(4 \pi)^{-1} \int \mathrm{du}$ is denoted by \langle\rangle (and a similar prescription holds for the primed variable). The rods have length $L$ and diameter $D$. It is convenient to scale the number density $\rho$ by the excluded volume $(\pi /$ 4) $L^{2} D$; viz., $c \equiv(\pi / 4) L^{2} D \rho$. Note that the second virial approximation is legitimate for low volume fractions: $D c / L$ $\ll 1$. Hence, the binary term can be appreciable when $c$ $=O(1)$ even though higher order terms remain negligible in that case. But then the second virial approximation is realistic for very slender rods only $(L \gtrsim 20 D)$. We remark that the solution remains isotropic up to $c^{*}=3.2904$ as calculated accurately by numerical analysis. 8,9

Usually one would approximate the unknown function $f(\mathbf{k}, \mathbf{u})$ in eq 2 and insert this into eq 1 . However, although the kernel in the integral equation is nondefinite, it is possible to attack eqs 1 and 2 in one go. A generalization of the Schwinger variational principle to nondefinite operators has been attributed to Selengut and Rowlands in the field of neutron transport theory (see Appendix B to ref 10 ). Bearing in mind that the kernel in eq 2 is symmetric, we apply their result to eqs 1 and 2

$$
\begin{aligned}
S_{\mathbf{v}}(\mathbf{k})= & S_{\mathbf{v}}[\varphi(\mathbf{k}, \mathbf{u})]=\left\langle s_{\mathbf{k}}(\mathbf{u}) \varphi(\mathbf{k}, \mathbf{u})\right\rangle^{2} /\left[\left\langle\dot{\varphi}^{2}(\mathbf{k}, \mathbf{u})\right\rangle+\right. \\
& \left.\frac{8 c}{\pi}\left\langle\left\langle s_{\mathbf{k}}(\mathbf{u}) \varphi(\mathbf{k}, \mathbf{u}) \sin \gamma\left(\mathbf{u}, \mathbf{u}^{\prime}\right) s_{\mathbf{k}}\left(\mathbf{u}^{\prime}\right) \varphi\left(\mathbf{k}, \mathbf{u}^{\prime}\right)\right\rangle\right\rangle^{\prime}\right]
\end{aligned}
$$

The actual structure factor $S(\mathbf{k})$ is the extremum of $S_{\mathrm{v}}(\mathbf{k})$ as the trial function $\varphi(\mathbf{k}, \mathbf{u})$ is varied; i.e., $S(\mathbf{k})=S_{\mathrm{v}}[f(\mathbf{k}, \mathbf{u})]$. It is stressed that variational principles of the Schwinger type can be quite powerful since in their derivation the trial function has been varied twice. This is exemplified in polymer dynamics, where primitive trial functions used in similar principles meet with considerable success. ${ }^{1-4}$ Here again, our very crude choice $\varphi(\mathbf{k}, \mathbf{u})=s_{\mathbf{k}}(\mathbf{u})$ will be shown to give accurate results. ${ }^{14}$ This choice has some merit since it is approximately valid for very low densities or very high values of the dimensionless quantity $L k$.

$$
\begin{aligned}
& S(\mathbf{k}) \simeq S_{\mathbf{v}}\left[s_{\mathbf{k}}(\mathbf{u})\right]= \\
& \frac{\left\langle s_{\mathbf{k}}{ }^{2}(\mathbf{u})\right\rangle^{2}}{\left\langle s_{\mathbf{k}}{ }^{2}(\mathbf{u})\right\rangle+\frac{8 c}{\pi}\left\langle\left\langle{s_{\mathbf{k}}}^{2}(\mathbf{u}) \sin \gamma{s_{\mathbf{k}}}^{2}\left(\mathbf{u}^{\prime}\right)\right\rangle\right\rangle^{\prime}}
\end{aligned}
$$

Letting $\mathbf{K}$ be the $z$ axis, we introduce polar coordinates $(\theta, \varphi)$ with $x \equiv \cos \theta$ and rewrite the average involving sin $\gamma$

$$
\begin{aligned}
& \left\langle\left\langle s_{\mathbf{k}}{ }^{2}(\mathbf{u}) \sin \gamma s_{\mathbf{k}}{ }^{2}\left(\mathbf{u}^{\prime}\right)\right\rangle\right\rangle^{\prime}= \\
& \quad \frac{1}{4} \int_{-1}^{1} \mathrm{~d} x \int_{-1}^{1} \mathrm{~d} x^{\prime}{s_{\mathbf{k}}}^{2}(x){s_{\mathbf{k}}}^{2}\left(x^{\prime}\right)\left(\frac{1}{2 \pi} \int_{0}^{2 \pi} \mathrm{d} \varphi \sin \gamma\right)
\end{aligned}
$$

The $\varphi$ integration is effected by expanding $\sin \gamma$ in terms of even Legendre polynomials and using the addition theorem. Here, we need retain the first two terms only

$$
\frac{1}{2 \pi} \int_{0}^{2 \pi} \mathrm{d} \varphi \sin \gamma=\frac{\pi}{4}-\frac{5}{32} \pi P_{2}(x) P_{2}\left(x^{\prime}\right)-\ldots
$$

Accordingly, our approximation for $S(\mathbf{k})$ becomes

$$
S(\mathbf{k}) \equiv S(K, c)=\frac{F^{2}(K)}{F(K)+2 c F^{2}(K)-\frac{5}{4} c G^{2}(K)}
$$

where the single-rod form factor is given by

$$
F(K) \equiv\left\langle s_{\mathbf{k}}{ }^{2}(\mathbf{u})\right\rangle=K^{-1} \mathrm{Si}(2 K)-\left(\frac{\sin K}{K}\right)^{2}
$$

and a first-order correction term is denoted by

$$
G(K)=\frac{3}{4} K^{-2}\left(1-\frac{\sin 2 K}{2 K}\right)-\frac{1}{2} F(K)
$$

If $G$ is set equal to zero in eq 8 , we regain the "conventional" random-phase approximation which is the zero-order result in the calculations by Shimada et al. ${ }^{5}$ But our higher order terms differ from theirs because the respective approximation schemes are not the same.

Equation 8 is in very good agreement with the numerical work of Maeda. ${ }^{7}$ In Table I we compare numerical with analytical values of the quantity $1+2 c-\bar{S}^{-1}(K)$, where $\bar{S} \equiv S / F$ and we have subtracted the trivial term $1+2 c$ 
Table I

Values of the Quantity $1+2 c-\bar{S}^{-1}(K)$ from the Numerical

Work of Maeda ${ }^{7}$ Compared with Those from Eq 8 with $G$ Neglected and $G$ Taken into Account

\begin{tabular}{ccccc}
\hline & & & \multicolumn{2}{c}{ eq 8} \\
\cline { 3 - 5 } scaled density & $k L=2 K$ & numerical & $G=0$ & $G \neq 0$ \\
\hline$c=0.04$ & 1 & 0.0022 & 0.0022 & 0.0022 \\
& 2 & 0.0083 & 0.0082 & 0.0083 \\
& 4 & 0.0272 & 0.0262 & 0.0272 \\
& 8 & 0.0542 & 0.0514 & 0.0540 \\
$c=0.4$ & 15 & 0.0661 & 0.0640 & 0.0659 \\
& 1 & 0.0218 & 0.0218 & 0.0218 \\
& 2 & 0.0831 & 0.0822 & 0.0830 \\
& 4 & 0.2721 & 0.2621 & 0.2716 \\
$c=4$ & 8 & 0.5417 & 0.5138 & 0.5404 \\
& 15 & 0.6612 & 0.6399 & 0.6590 \\
& 1 & 0.2318 & 0.2176 & 0.2182 \\
& 2 & 0.8745 & 0.8216 & 0.8304 \\
& 4 & 2.7961 & 2.6208 & 2.7161 \\
& 8 & 5.4337 & 5.1384 & 5.4037 \\
& 15 & 6.6174 & 6.3992 & 6.5896
\end{tabular}

$=\bar{S}^{-1}(0)$ related to the osmotic compressibility.

It is important to note that $\bar{S}(K)$ is a monotone increasing function of $K$. This implies that the oscillations in $\bar{S}(K)$ found in experiments ${ }^{11,12}$ on TMV and fd virus in salt-free solutions can be explained only by incorporating third-order and higher order virial terms into the present theory. A stringent test of the structure factor derived here and in refs $5-7$ requires experiments on very slender rods like schizophyllan and fd virus (but in excess salt) for which the second virial approximation is more readily justified.

\section{References and Notes}

(1) Fixman, M. J. Chem. Phys. 1982, 76, 6124.

(2) Fixman, M. J. Chem. Phys. 1983, 78, 1588.

(3) Odijk, T. Macromolecules 1986, 19, 2073.

(4) Doi, M.; Edwards, S. F. The Theory of Polymer Dynamics; Clarendon: Oxford, 1986.

(5) Shimada, T.; Doi, M.; Okano, K. J. Chem. Phys. 1988, 88, 2815.

(6) Doi, M.; Shimada, T.; Okano, K. J. Chem. Phys. 1988, 88, 4070.

(7) Maeda, T. Macromolecules 1989, 22, 1881.

(8) Kayser, R. F.; Raveché, H. J. Phys. Rev. A 1978, A17, 2067.

(9) Lekkerkerker, H. N. W.; Coulon, P.; van der Haegen, R.; Deblieck, R. J. Chem. Phys. 1984, 80, 3427.

(10) Tait, J. H. Neutron Transport Theory; Longmans: London, 1964.

(11) Maier, E. E.; Schulz, S. F.; Weber, R. Macromolecules 1988, 21 , 1544.

(12) Schulz, S. F.; Maier, E. E.; Weber, R. J. Chem. Phys. 1989, 90, 7.

(13) For a discussion of this point, see: van der Schoot, P.; Odijk, T. J. Chem. Phys., in press.

(14) Using a variational principle together with a trial function independent of variational parameters may almost appear a contradion in terms. But for a Schwinger principle it so happens that it is not. Indeed, its derivation proceeds in two stages (see, e.g., ref 10): (a) a first principle for $S(\mathbf{k})$ is written in terms of a trial function $\chi(\mathbf{k}) ;(\mathbf{b}) \chi(\mathbf{k})$ is set equal to $C(\mathbf{k})$. $\varphi(\mathbf{k})$, where the variational parameter $C(\mathbf{k})$ is obtained by minimizing or maximizing $S$. In our calculation the choice $\varphi(\mathbf{k})$ $=s_{\mathbf{k}}(\mathbf{u})$ amounts to $\chi(\mathbf{k})=C(\mathbf{k}) \mathbf{s}_{\mathbf{k}}(\mathbf{u})$, which is of course a nontrivial trial function.

\section{Communications to the Editor}

\section{Synthetic Glycoconjugates: Simple and Potential Glycoprotein Models Containing Pendant $N$-Acetyl-D-glucosamine and $\boldsymbol{N}, N^{\prime}$-Diacetylchitobiose}

Oligosaccharide chains of glycoconjugates are important biopolymers not only as carriers of information in cellcell interactions but as markers of cellular differentiation, aging, and malignant alteration. Synthetic carbohydrate polymers having pendant sugar residues are of great interest as artificial glycoconjugates from biochemical and medical aspects. In fact, as a result of increasing needs for synthetic carbohydrate polymers for medical use, there have been several examples of the preparation of sugaracrylamide copolymers. ${ }^{1-4}$

To mimic and utilize the unique molecular recognition nature of oligosaccharide chains effectively, our attention is now directed to the design and preparation of new types of "pseudoglycoproteins" containing the appropriate spacerarm structure between the sugar moiety and the main chain of copolymers. Pseudoglycoproteins as simple models of glycoproteins will provide simplified and useful information about complex functions of the saccharide region. Moreover, the introduction of a suitable spacer-arm moiety will permit flexibility of sugar side chains and thereby
Table I

Polymerizations of Carbohydrate Monomers and Acrylamide

\begin{tabular}{crccccc}
\hline $\begin{array}{c}\text { carbohydr } \\
\text { monomer }\end{array}$ & $\begin{array}{c}\text { monomer } \\
\text { ratio }^{a}\end{array}$ & $\begin{array}{c}\text { total } \\
\text { yield, } \\
\%\end{array}$ & $\begin{array}{c}\text { polym } \\
\text { compos }^{a}\end{array}$ & $\begin{array}{c}\text { sugar } \\
\text { content, } \\
\text { wt \% }\end{array}$ & $\begin{array}{c}{[\alpha]_{\mathrm{D}},} \\
\mathrm{deg}\end{array}$ & $\begin{array}{c}\eta_{\text {inh, }}{ }^{6} \\
\mathrm{dL} / \mathbf{g}\end{array}$ \\
\hline $\mathbf{6}$ & $1: 4$ & 46.1 & $1: 12$ & 23.5 & -6.2 & 0.27 \\
$\mathbf{6}$ & $1: 10$ & 57.4 & $1: 42$ & 8.0 & -4.1 & 1.15 \\
$\mathbf{7}$ & $1: 4$ & 61.9 & $1: 9$ & 29.6 & -9.6 & 0.43 \\
$\mathbf{7}$ & $1: 10$ & 59.0 & $1: 31$ & 11.0 & -4.5 & 1.26 \\
8 & $1: 4$ & 65.8 & $1: 8$ & 34.6 & -11.4 & 0.34 \\
$\mathbf{8}$ & $1: 10$ & 75.6 & $1: 28$ & 12.5 & -7.6 & 1.03 \\
$\mathbf{9}$ & & & & & & \\
$\mathbf{1 2}$ & $1: 4$ & 54.8 & $1: 8$ & 46.1 & -11.9 & 0.31 \\
$\mathbf{1 2}$ & $1: 10$ & 76.0 & $1: 23$ & 23.1 & -6.4 & 1.02
\end{tabular}

${ }^{a}$ Ratio of carbohydrate monomer and acrylamide. ${ }^{b}$ In $\mathrm{H}_{2} \mathrm{O}$ at 25 ${ }^{\circ} \mathrm{C}$. ${ }^{\circ}$ Insoluble in water.

accessibility to the active sites, the regulation of the contents of carbohydrate monomers, and molecular weights of the polymers. This paper deals mainly with a simple and convenient method for the preparation of carbohydrate monomers based on amino sugars closely related to the invariant "core" structure of N-linked type glycoproteins with an effective spacer-arm function. 